\title{
Cognitive changes in patients with epilepsy identified through the MoCA test during neurology outpatient consultation
}

DOI: https://doi.org/10.1016/i.yebeh.2021.108158

\section{Authors}

J.M. Montaño-Lozada, Norman López, L.M. Espejo-Zapata, Marcio Soto-Añari, Miguel Ramos-Henderson, Nicole Caldichoury-Obando, Loida Camargo

\section{Abstract}

Introduction

Epilepsy is a chronic neurological disorder that may occur alongside cognitive changes, with effects on multiple cognitive domains.

\section{Objective}

To compare the cognitive performance of patients with epilepsy and healthy controls through Montreal Cognitive Assessment (MoCA) during outpatient consultation at a reference diagnostic center in Colombia and analyze and the influencing factors.

Materials and methodology

One-hundred and four patients during neurology outpatient consultation in the city of Cartagena, Colombia, were assessed with the (MoCA) test, i.e., 54 people who consulted for headache and have not been diagnosed with epilepsy (NEP) and 50 with a diagnosis of epilepsy (EPs) according to the diagnostic criteria of the International League Against Epilepsy (ILAE).

\section{Results}

Significant differences were found in the total mean scores of the (MoCA) between (EPs) and (NPE) groups $(t=4.72 ; p<0.01)$, particularly in attention $(t=3.22 ; p<0.02)$ and memory $(t=5.04 ; p<0.01)$ dimensions. Additionally, a significant association was observed between years of schooling and (MoCA) scores $(p=0,019)$ but not between socioeconomic level $(p=0,510)$, age $(p=0,452)$ and the frequency of seizures $(p=0,471)$. 


\section{Discussion}

Patients with epilepsy show lower scores in several cognitive domains in respect of the control group. The (MoCA) has proven its appropriateness for cognitive screening in the contexts of clinical neurology outpatient consultation.

\section{Keywords}

Epilepsy, Short cognitive tests, (MoCA) Test, Cognitive dysfunction, Mental impairment 